\title{
Fatal Complications after Pediatric Surgical Interventions: Lessons Learned
}

\author{
Willemijn M. Klein ${ }^{1}$ Mayke E. Van der Putten ${ }^{2}$ Benno Kusters ${ }^{3}$ Bas H. Verhoeven ${ }^{4}$
}

\footnotetext{
${ }^{1}$ Department of Radiology and Nuclear Medicine, Radboud University Medical Center, Nijmegen, The Netherlands

2 Department of Neonatology, Radboud University Medical Center, Nijmegen, The Netherlands

${ }^{3}$ Department of Pathology, Radboud University Medical Center, Nijmegen, The Netherlands

${ }^{4}$ Department of Pediatric Surgery, Radboud University Medical Center, Nijmegen, The Netherlands
}

Eur J Pediatr Surg Rep 2017;5:e12-e16.
Address for correspondence Willemijn M. Klein, MD, PhD, Department of Radiology and Nuclear Medicine, Radboud University Medical Center, Geert Grooteplein Zuid 10, Nijmegen 6525GA, The Netherlands (e-mail: willemijn.klein@radboudumc.nl).
Abstract
Keywords
- ventriculoperitoneal shunt
- unexpected death
- complications
- feeding tube
- chest drain

Placement of catheters, drains, shunts, and tubes in children can lead to serious or even fatal complications at the moment of placement, such as hemorrhage at insertion, or in the longterm, such as infections and migration into adjacent organs. The clinician should always be aware of these potential complications, especially if the child is unwell. For postmortem diagnostic evaluation, either with a computed tomography scan or an invasive autopsy, all tubes, drains, shunts, and/or catheters should be left in situ. We present three cases with fatal complications after the placement of a chest drain, ventriculoperitoneal shunt, and gastrostomy tube.

\section{New Insights and the Importance for the Pediatric Surgeon}

In cases of unexpected death, one should be attentive to complications of the catheters and drains. Postmortem radiology can be used complementary or as an alternative to conventional autopsy.

\section{Introduction}

Indwelling devices such as catheters, drains, shunts, and tubes are commonly used in the treatment of acute and chronic patients and by different specialities. Surgeons/ doctors placing the device and caregivers looking after it have to realize that its use can potentially lead to serious complications or even death. Complications can occur at any time during and after implantation. Approximately 15\% of adults with central venous catheters (CVC) are known to have complications. ${ }^{1,2}$ In pediatric patients, adverse events with CVCs may cause complications in up to $46 \%$ of cases. ${ }^{3}$ The placement and presence of CVC can be complicated by arterial puncture, hemorrhage, hemothorax, pneumothorax, visceral or nerve injury, cardiac tamponade, air embolism, arrhythmia, (intraperitoneal) migration, dislocation, infec- tion, thrombosis, and pulmonary emboli. Chest drain insertion is commonly performed in trauma patients. A retrospective study among adult trauma patients in South Africa showed that $19 \%$ are associated with complications ( $2 \%$ visceral and $1 \%$ vascular injuries). ${ }^{4}$ Complications of chest drains in neonates, especially prematures, are rare. Some cases in literature report thoracic duct perforation and lung transection. ${ }^{5,6}$

Gastrostomies are known to have a high percentage (25-50\%) of complications, depending on the technique used and definition of minor and major complications. ${ }^{7-10}$ After maturing of the channel, malposition of the tube is rare. Ventriculoperitoneal shunts (VPSs) and drains can luxate, can cause infections, or can migrate into organs,thus causing complications. The overall complication rate of VPS is very received

May 20, 2016

accepted after revision

January 26, 2017
DOI http://dx.doi.org/

$10.1055 / \mathrm{s}-0037-1599795$

ISSN 2194-7619. (c) 2017 Georg Thieme Verlag KG Stuttgart • New York
License terms

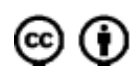


high (50\%), mainly including infection and obstruction. ${ }^{11}$ We present three cases with fatal outcomes after placement of a drain, a tube, and a shunt. In all three cases, postmortem radiology and/or conventional autopsy were performed to analyze the fatal events and to establish the cause of death.

\section{Case 1}

A male neonate was born with a (prenatally diagnosed) large left-sided diaphragmatic herniation that included the stomach, spleen, jejunum, and part of the colon and the liver. The boy was intubated immediately postpartum; however, he had continuous problems with oxygenation and ventilation caused by persistent pulmonary hypertension (PPHN), and consequently venoarterial extracorporeal membrane oxygenation (VA-ECMO) was started with the use of the right jugular vein and the carotid artery. The boy was stabilized, and at 5 days of age, the diaphragm, as well as the abdominal wall,was surgically closed with Gore-Tex patches. This procedure was complicated by a small rupture of the splenic capsule for which the superior vascular segment was devascularized by sealing the short gastric branches and the upper branch of the splenic artery. There were no further complications in the following postoperative days, and therefore on day 8, the VA-ECMO cannulas were removed. Within a few days following a respiratory infection, rebound PPHN occurred and caused progressive oxygenation and ventilation problems. Blood cultures were negative, but pleural fluid was positive for Parabacteroides distasonis. High-frequency oscillation, nitric oxide, inotropics, and milrinone resulted in some improvement, as did drainage of a pleural effusion on the left side with the Turkel Safety thoracentesis and paracentesis system of Covidien (diameter $2.7 \mathrm{~mm}$ [8 Fr], length $12.7 \mathrm{~cm}$; Medtronic, Mansfield, Massachusetts) (-Fig. 1). This system has a blunt core with a sharp needle surrounding this core. The moment the resistance is lost (intrathoracic), the blunt core appears again and protects the lung. A day later, the situation deteriorated with primarily low saturations between 50 and $80 \%$. A chest radiograph showed pleural effusion on the right side, and,

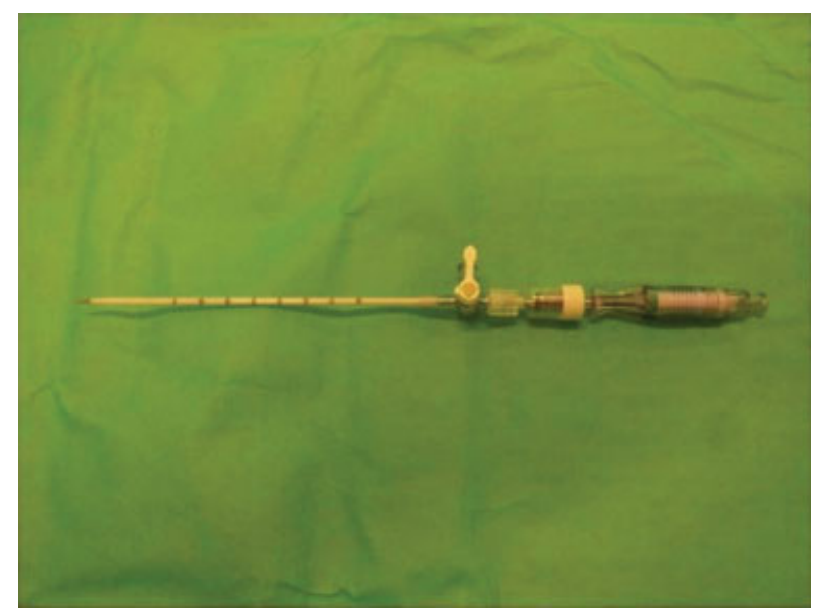

Fig. 1 Turkel Safety thoracentesis and paracentesis system of Covidien. as a last attempt, despite the expectation of only marginal improvement, another chest drain (same type) was placed. While placing the chest drain, fresh blood came out of the drain. Without any further alternative treatment options, the boy died within minutes. The parents consented to postmortem imaging but not an autopsy or any other invasive procedure.

The postmortem computed tomography (CT) and magnetic resonance imaging (MRI) demonstrated a hydropic neonate with a right-sided chest drain inserted between the secondand thirdribs, with an interpleural course and the tip in between the large mediastinal vessels apical to the aortic arch ( Fig. 2). There was a large interpleural hemorrhage as well as peritoneal hemorrhage. The right lung was atelectatic with intrapulmonary signs of a previous hemorrhage and a recent interpleural hemorrhage. The hypoplastic left lung was atelectatic with pleural effusion and a pneumothorax. The tip of the left chest drain was positioned paramediastinal. The spleen also showed signs of recent rupture and bleeding.

To summarize, the boy died after complications due to congenital diaphragmatic herniation and rebound PPHN, with fatal hemorrhage after the insertion of the right-sided chest drain.

\section{Case 2}

A 10-year-old girl who was born prematurely at 28 weeks of gestation with a spina bifida aperta at L4, which was corrected shortly after birth. The hydrocephalus was corrected with a VPS. She performed continuous intermittent catheterization and used a wheelchair, but otherwise she had a medically uneventful childhood. One day she had fever and vomiting, but her parents did not consult a doctor. Two days later, she became unwell and had a cardiac arrest at home. Ambulance staff commenced resuscitation and brought her into our emergency department. The girl was asystolic, had unresponsive pupils, and died 70 minutes after the start of resuscitation. The parents consented to postmortem imaging and full-body autopsy.

The postmortem CT demonstrated that the VPS was located intraperitoneal, however, entering the jejunum, with pneumatosis of the intestine, and peritoneal gas and fluid, indicating bowel perforation and peritonitis. This was confirmed at autopsy, which also discovered that the entry of the VPS was in the proximal jejunum and the perforation in the distal jejunum; further necrotic bowel walls and extensive peritonitis (-Fig. 3). There were no signs of meningitis. The cause of death was determined to be due to peritonitis after migration of the VPS in the jejunum, with bowel ischemia and perforation.

\section{Case 3}

A 7-year-old girl suffered from severe retardation and symptomatic epilepsy caused by cerebro-oculo-cutaneous syndrome. She had feeding difficulties and therefore received a percutaneous endoscopic gastrostomy (PEG) at the age of 7 years. Five months after placement of the PEG, the PEG was 

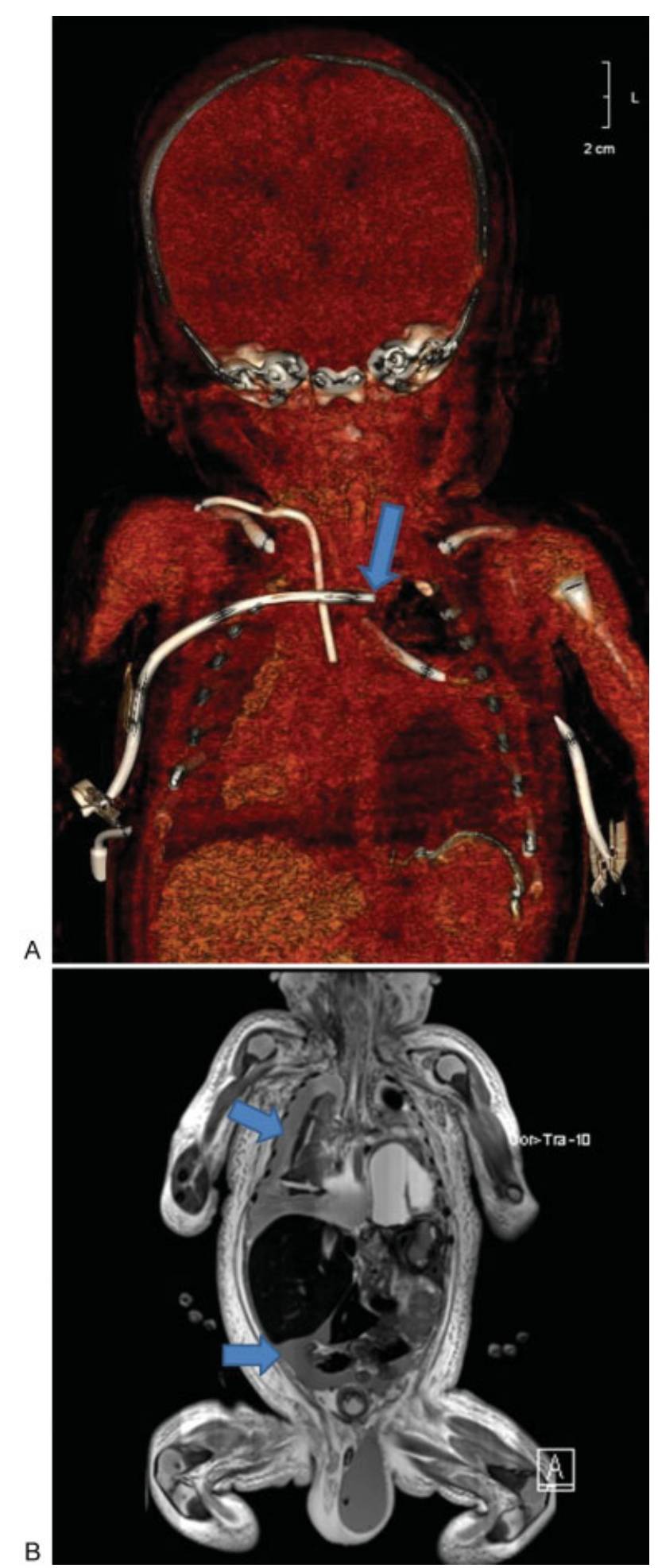

Fig. 2 A male neonate with a corrected left-sided congenital diaphragmatic hernia. The right-sided chest drain was malpositioned through the mediastinum and caused a fatal hemorrhage. (A) The postmortem computed tomography and three-dimensional reconstruction shows the right-sided chest drain transversing the mediastinum (arrow). (B) The postmortem magnetic resonance imaging demonstrates the extensive intrapleural and intraperitoneal hemorrhage (arrows).

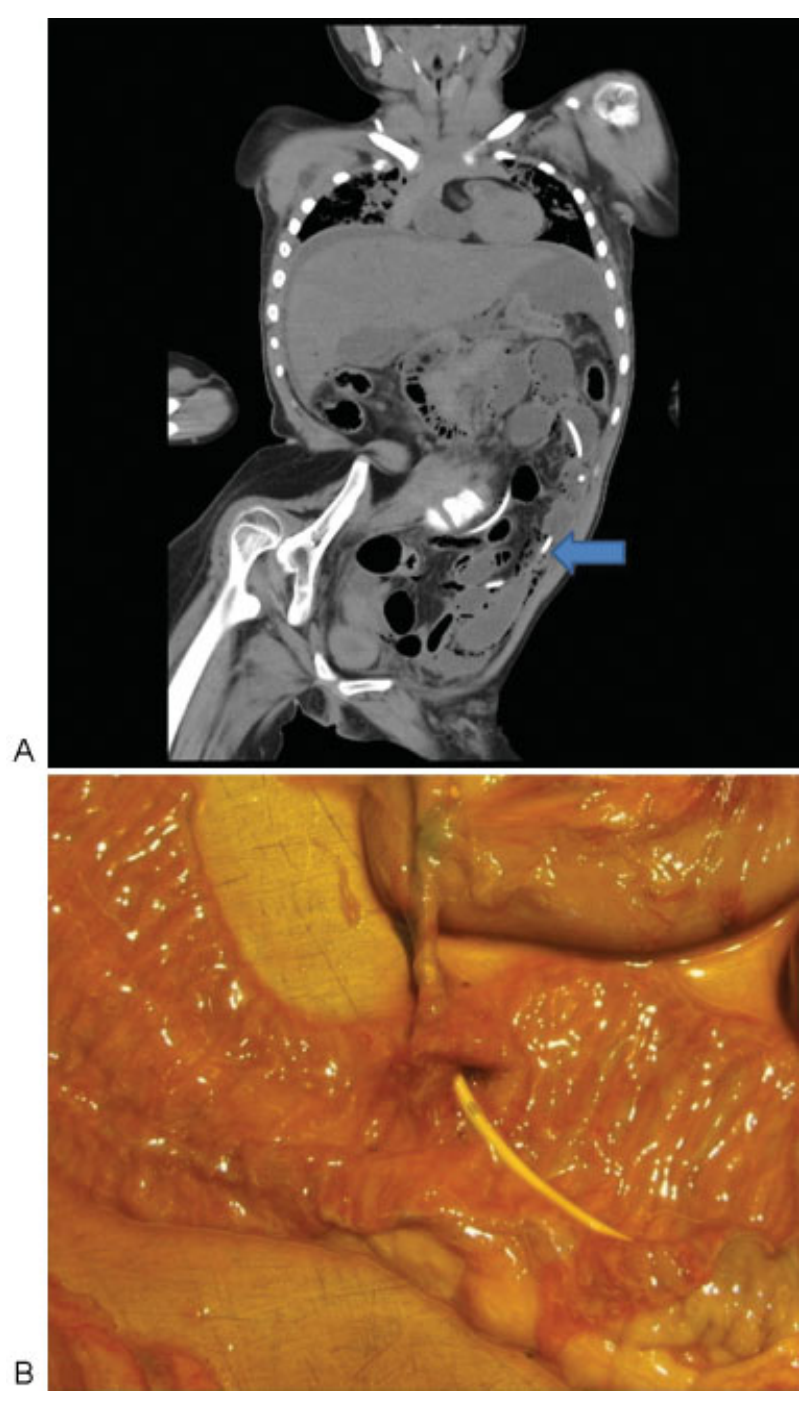

Fig. 3 A 10-year-old girl with spina bifida aperta. The hydrocephalus was corrected with a ventriculoperitoneal shunt (VPS). The girl died after the shunt had migrated into the jejunum and had caused bowel ischemia, perforation, and peritonitis. The postmortem computed tomography(A) and the autopsy image (B) show the VPS in intraluminal jejunal location.

removed and a Flocare Gastro-Tube Ch 14 (Nutricia) was placed under gastroscopic surveillance. The gastrostomy appeared to run oblique through the abdominal wall. The replacement was without problems. One week later, the gastrotube was replaced uneventfullyby a MIC-KEY Ch 14 2.3-cm feeding tube (Klinboley Clash). Shortly after her eighth birthday (15 months after the initial PEG), the MICKEY luxated when taking off her clothes. It was reinserted at the emergency department of our hospital after several hours of delay by an experienced pediatric surgeon who replaced 150 buttons or more. The channel had to be cannulated and dilated with a Ch 12 and 14 silicone Foley catheter. Gastric content was aspirated, after which the catheter was changed for a new MIC-KEY Ch 14 2.3-cm feeding tube. We performedneither a new aspiration of gastric content after placement of the MIC-KEY nor a contrast 
study, as the initial gastric content aspiration indicated correct placement. The girl was discharged after an hour without pain or discomfort and with clear instructions to the parents. In the following days, the tube tract seemed slightly painful. Parents gave medication and tube feeding without problems or complaints. After 48 hours of placement of the MIC-KEY, the girl was found dead in her bed while lying in vomit. The parents consented to postmortem CT and full body autopsy. The CT demonstrated a malposition of the MIC-KEY just outside the stomach, with extensive intraperitoneal free gas and fluid with high radiodensity suggestive of perforation and leakage of enteral feeding and peritonitis. (- Fig. 4) Autopsy confirmed these findings as well as a perforation in the skin-to-stomach fistula. Food and medicines were found in the peritoneal cavity with diffuse peritonitis. The cause of death was peritonitis due to MICKEY gastrostomy feeding tube perforation.

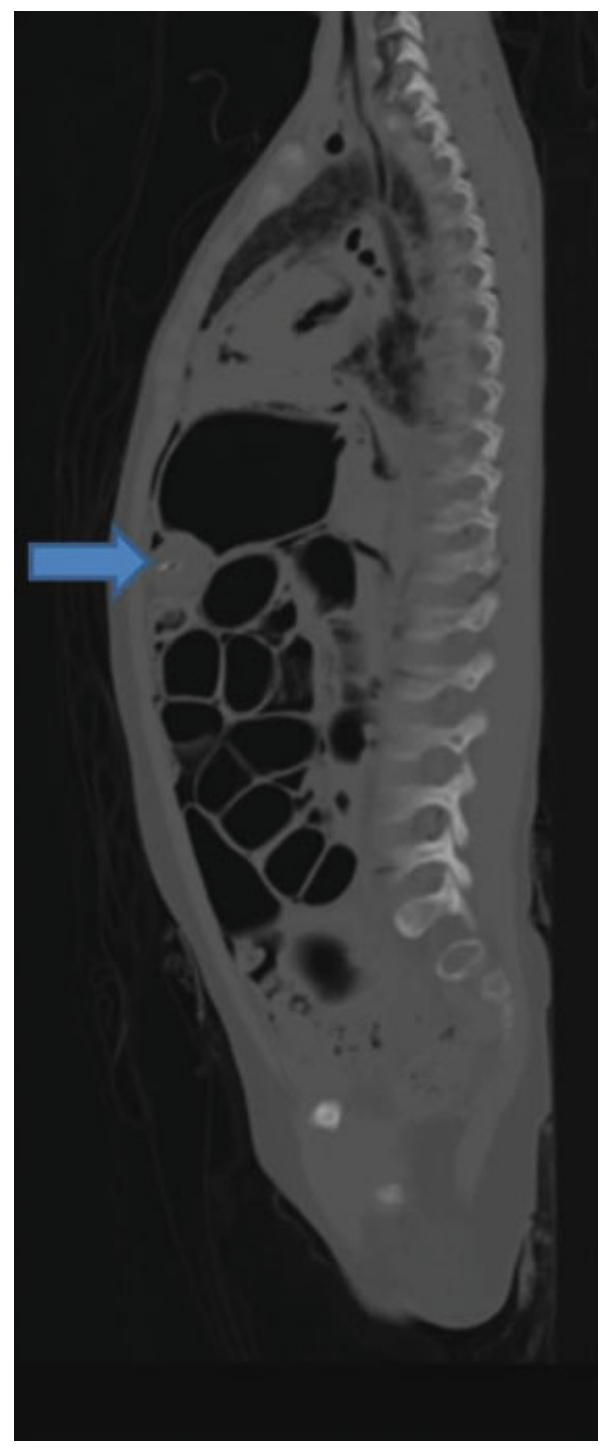

Fig. 4 An 8-year-old girl died of peritonitis after malpositioning of a gastrostomy feeding tube into the peritoneal cavity. The postmortem computed tomography demonstrates the balloon of the MIC-KEY just outside the stomach (arrow).

\section{Discussion}

With these three cases, we demonstrated fatal complications after placement of catheters or drains, either immediately or in the short- or the long-term period. The causes of death were diagnosed by postmortem CT and autopsy. All three cases were reported to the inspecting authority and judged to be complications. Root cause analysis was performed in all three cases.

In the first case, a chest drain was placed; however, the malpositioning caused an immediate fatal hemorrhage. Subcutaneous edema may have led to misjudgment as to the correct insertion location as well as the length of and the scale on the thoracic drain, which starts at the last side hole and not at the tip. The malposition of the chest drain and the extensive hemorrhage were demonstrated on postmortem CT and MRI. Unfortunately, the parents did not consent to further postmortem CT angiography or autopsy. As mentioned, complications of chest drains in neonates, especially prematures, are rare. There are some cases in literature reporting thoracic duct perforation and lung transaction. ${ }^{5,6}$ Pigtail catheters seem to be a safer alternative. ${ }^{12}$ We found no reports on fatal hemorrhage.

Bowel perforation from VPS is an extremely rare but serious complication. It is reported to occur in less than $0.1 \%$ of cases, usually 2 to 20 months after insertion and often with anal protrusion of the shunt. Peritonitis or meningitis (with enteric bacteria) can occur in $25 \%$ of cases; however, generally, cases are asymptomatic or unrecognized. It has a high mortality rate of $15 \%{ }^{13}$ In the case we presented, the subtle signs of fever in a retarded girl did not alert the parents to consult a doctor or visit a hospital.

Major complications of gastrostomies occur not only directly after primary placement (gastrointestinal perforation malpositioning of the tube and gastrocolic fistula formation) but also in the long run (mainly peristomal infections). ${ }^{14,15}$ In the presented case, the peritonitis was due to a malposition of MIC-KEY after reinsertion. The fistula between the skin and the stomach was perforated with the blunt tip of MIC-KEY. This is a very rare complication when replacement is done later than 2 months after initial PEG placement. ${ }^{16}$ Intraperitoneal placement after 90 days has been described by Aprahamian et al after U-stitch laparoscopic gastrostomy (1.1\%). ${ }^{17}$ Normally, it is discovered immediately after replacement or after administering feeding or medications. This patient suffered a severe intellectual disability that made it difficult for her to indicate pain or feeling of illness, which may have been misleading for the parents and consulted clinician.

To prevent the described complications in the future, we suggest to provide more education with regard to the materials used (such as a scale on the drain) and make use of imaging during or after placement. In many cases, ultrasound or (contrast)radiography can be used. After placement, lowthreshold control of the position of shunt and drains is advised especially in patients who are not well. The hospital protocols have been changed according to our findings and advices. 
Klein et al.

In these three cases, postmortem CT clearly showed the malposition of the drains and tubes. Autopsy provided more details on the exact location of the migration of the VPS in the jejunum and on the location of the perforation in case 2 . In case 3 , autopsy was able to demonstrate the fausse route in the gastrostomy fistula wall that was not visible on postmortem CT. With the declining numbers of autopsies over the past half century, an increase in postmortem radiology (virtual autopsy) over the last decade has been observed. Postmortem CT and MRI are used not only in forensic cases of unnatural death but also in cases of assumed natural death. ${ }^{18}$ In the presented cases, a contrast agent through the chest drain, VPS, and MIC-KEY would have given more information on the location of malpositioning. Postmortem CT angiography could have been a useful addition to demonstrate the site of the fatal hemorrhage in case 1 due to the high suspicion of vascular damage near the tip of the chest drain. Contrast agent administration is advised in cases where an autopsy was refused. We emphasize the importance of leaving all catheters, drains, and tubes in place for postmortem investigations, even when clinically there is no indication of malpositioning.

\section{Conclusion}

Children with catheters and drains have a risk of complications that can be unexpected and fatal. Symptoms can be subtle or occult, especially in children with intellectual impairment or sedation. Most caregivers know the potential complications and act accordingly, but major complications unfortunately happen. After death, we advise to always leave all catheters and drains in situ for postmortem diagnostic evaluation, both with virtual or conventional autopsy. Learning from these events is essential, and modern (postmortem) investigations can help to find the cause and prevent calamities, as described, in future.

\section{Conflict of Interest}

None.

\section{References}

1 McGee DC, Gould MK. Preventing complications of central venous catheterization. N Engl J Med 2003;348(12):1123-1133

2 Eisen LA, Narasimhan M, Berger JS, Mayo PH, Rosen MJ, Schneider RF. Mechanical complications of central venous catheters. J Intensive Care Med 2006;21(01):40-46
3 Bradford NK, Edwards RM, Chan RJ. Heparin versus 0.9\% sodium chloride intermittent flushing for the prevention of occlusion in long term central venous catheters in infants and children: a systematic review. Int J Nurs Stud 2016;59:51-59

4 Kong VY, Oosthuizen GV, Sartorius B, Keene C, Clarke DL. An audit of the complications of intercostal chest drain insertion in a high volume trauma service in South Africa. Ann R Coll Surg Engl 2014; 96(08):609-613

5 Kumar SP, Belik J. Chylothorax-a complication of chest tube placement in a neonate. Crit Care Med 1984;12(04):411-412

6 Brooker RW, Booth GR, DeMello DE, Keenan WJ. Unsuspected transection of lung by pigtail catheter in a premature infant. J Perinatol 2007;27(03):190-192

7 Goldin AB, Heiss KF, Hall M, et al. Emergency department visits and readmissions among children after gastrostomy tube placement. J Pediatr 2016;174:139-145.e2

8 Liu R, Jiwane A, Varjavandi A, et al. Comparison of percutaneous endoscopic, laparoscopic and open gastrostomy insertion in children. Pediatr Surg Int 2013;29(06):613-621

9 Baker L, Beres AL, Baird R. A systematic review and meta-analysis of gastrostomy insertion techniques in children. J Pediatr Surg 2015;50(05):718-725

10 Dookhoo L, Mahant S, Parra DA, John PR, Amaral JG, Connolly BL. Peritonitis following percutaneous gastrostomy tube insertions in children. Pediatr Radiol 2016;46(10):1444-1450

11 Zhao R, Shi W, Yu J, Gao X, Li H. Complete intestinal obstruction and necrosis as a complication of a ventriculoperitoneal shunt in children: a report of 2 cases and systematic literature review. Medicine (Baltimore) 2015;94(34):e1375

12 Wei YH, Lee $\mathrm{CH}$, Cheng HN, Tsao LT, Hsiao CC. Pigtail catheters versus traditional chest tubes for pneumothoraces in premature infants treated in a neonatal intensive care unit. Pediatr Neonatol 2014;55(05):376-380

13 Ghritlaharey RK, Budhwani KS, Shrivastava DK, et al. Trans-anal protrusion of ventriculo-peritoneal shunt catheter with silent bowel perforation: report of ten cases in children. Pediatr Surg Int 2007;23(06):575-580

14 Landisch RM, Colwell RC, Densmore JC. Infant gastrostomy outcomes: the cost of complications. J Pediatr Surg 2016;51(12): 1976-1982

15 McSweeney ME, Kerr J, Jiang H, Lightdale JR. Risk factors for complications in infants and children with percutaneous endoscopic gastrostomy tubes. J Pediatr 2015;166(06):1514-9.e1

16 McSweeney ME, Jiang H, Deutsch AJ, Atmadja M, Lightdale JR. Long-term outcomes of infants and children undergoing percutaneous endoscopy gastrostomy tube placement. J Pediatr Gastroenterol Nutr 2013;57(05):663-667

17 Aprahamian CJ, Morgan TL, Harmon CM, Georgeson KE, Barnhart DC. U-stitch laparoscopic gastrostomy technique has a low rate of complications and allows primary button placement: experience with 461 pediatric procedures. J Laparoendosc Adv Surg Tech A 2006;16(06):643-649

18 Arthurs OJ, van Rijn RR. Paediatric and perinatal postmortem imaging: mortui vivos docent. Pediatr Radiol 2015;45(04): 476-477 